\title{
INNOVATIVE NURSING LEADERSHIP IN YOUTH HEALTH
}

Katherine M. Nelson, RN, PhD, Senior Lecturer, Graduate School of Nursing, Midwifery and Health, Victoria University of Wellington

Margaret Connor, RN, PhD, Research Fellow, Graduate School of Nursing, Midwifery and Health, Victoria University of Wellington

Gillian Alcorn, NP, MA (Applied) in Nursing, Youth Health Nurse Specialist, Vibe Youth Transition Service, Hutt Valley

\section{Abstract}

Along with introducing the New Zealand Primary Health Care Strategy the Ministry of Health funded 11 primary health care nursing innovation projects for three years. One of these was sited in the Hutt Valley District Health Board region at what was originally known as the Hutt Valley Youth Service and later named the Vibe service. The funding supported employment of a clinical nurse leader who later, during the course of the project, qualified as a Nurse Practitioner (NP). The dynamic leadership provided by the nurse extended beyond service boundaries benefiting policy decision making throughout the District Health Board region and contributing to youth health and service development nationally. In return for fostering such leadership Vibe gained access to new dimensions of youth health care. It became apparent that high calibre clinical leadership, a major component of the NP scope of practice in primary health care, improves outcomes for the clientele, increases satisfaction for the team members and provides a model for potential NPs wherever they are situated.

Key Words: Clinical leadership, innovation, youth health, nurse practitioner, primary health care.

\section{Introduction}

The New Zealand Primary Health Care Strategy (Ministry of Health, 2001) acknowledges the contribution of clinical leadership to positive outcomes for clients of primary health care $(\mathrm{PHC})$ facilities. Clinical leadership can emerge from any of the health disciplines in PHC. However, the Strategy vision and mission specifically recognises the potential contribution of nursing. Consistent with this vision, in 2003 the Ministry funded 11 nursing innovation projects and their evaluation. The Hutt Valley Youth Health Service (later named Vibe) was chosen to be one of these project sites. Vibe successfully advanced its remit during the project (Primary Health Care Nurse Evaluation Team, 2007). As the project aim and objectives concerned the development of a nurse position, success was tied intimately to the expanding sphere

Nelson, K. M., Connor, M., \& Alcorn, G. (2009). Innovative nursing leadership in youth health. Nursing Praxis in New Zealand, 25(1), 27-37. 
of influence of the nurse's clinical leadership ability. The nurse was on the cusp of becoming a Nurse Practitioner (NP) as she took up the position and registered in this scope during the project period. The NP scope of practice requires the demonstration of leadership (Nursing Council of New Zealand, 2004).

While there has been a considerable focus in New Zealand on leadership in nursing generally (Ministry of Health, 1995), recently the focus has moved more specifically to PHC nursing leadership (MacKay, 2002; Nursing and Midwifery Workforce Strategy Group, 2006). Developments in PHC nursing leadership internationally have focused on both clinical leadership (Abbott, 2007) and leadership in nurse-led multidisciplinary teams (Solheim, McElmurry, \& Kim, 2007). One writer reviewing international developments argues that clinical leadership and primary health care nursing go hand in hand (McMurray, 2007).

The purpose of this paper is to demonstrate how, with targeted innovation nursing funding, a nurse was employed to work in a multidisciplinary non-government organisation (NGO) youth health service in a clinical leadership role. The paper is written by two researchers involved with the Vibe site (KN, MC) from the evaluation team, and the nurse (GA) employed by Vibe. It draws on data gathered as part of the evaluation, and earlier and current Vibe sources. Evaluation data included interviews with the nurse and stakeholders, focus groups with service clientele and school nurses, and Vibe records. Vibe sources included internally commissioned evaluations, documents and service records.

The paper discusses how within the position created, the nurse increased the nursing sphere of influence through development of her leadership capacity. Co-locating a nurse with a brief to lead the development of a multidisciplinary service is atypical in the New Zealand PHC sector. Learning more about the "essence of the clinical nurse leader, the environment in which they operate or how and where the impact is felt" is necessary (Hyrkäs \& Dende, 2008, p. 497).

At the time of gaining innovation funding Vibe had a seven year history of youth health service delivery in the Hutt Valley. The Hutt Valley District Health Board (HVDHB), HVDHB Planning and Funding - Youth Health Portfolio Manager, Vibe Trust Board and management were committed to the development of Vibe as a model of best practice in youth health delivery. This shared vision of expanded youth health delivery both external to the service and within VIBE made a positive vehicle for outside funding to be realised. This environment provided a supportive structure in which the nurse's leadership potential was realised. The paper firstly provides some context and background to the Vibe service and the appointment of the nurse. It then proceeds to demonstrate the nurse's contribution to Vibe under two headings: practice development and service development. It concludes with some personal reflections from the nurse (GA) at Vibe. 


\section{Background and Context}

The Vibe Service began in Lower Hutt in 1996 in recognition of the special health needs of young people and their reluctance to attend mainstream services. The then Hutt Valley Health and Hospital service, now the HVDHB purchased a contract from Vibe for this service. Since that time, Vibe has expanded into the comprehensive multidisciplinary health service it is today. Vibe provides a free and confidential health and social support service for young people $(10-24$ years) and youth transition services for those aged $15-19$ years. The number of clients registered with Vibe (anyone who has used the service) increased from 3,243 in the middle of 2004 to 7,400 in 2008. These young people are reflective of the Hutt Valley youth population. Most identified as Pakeha with $28 \%$ identifying as Maori and $8 \%$ as Pacific people (Connor $\&$ Nelson, 2006).

The nurse began her role in 2003. At the time of appointment, there was no coherent policy structure governing clinical practice. Vibe directly employed a small number of peer support workers and a community youth worker and contracted a number of clinicians (nurses and medical practitioners) from other services. Peer support workers are a group of young people who take on a direct interactive role with the clientele after preparatory in-house training and support to undertake national youth worker qualifications. A youth development philosophy permeates all work with young people at Vibe and peer support workers' practice includes reception duties, one-on-one support, health promotion, community liaison and training concerning youth appropriate practice. It is believed that the young clientele will relate readily to these workers who are likely to experience and understand the context that brings them into the Vibe service. The contracted clinicians operated under their main employing service's policies and standards.

Registration as an NP without prescribing rights took place in December 2005. The nurse made an informed decision to invest personal energy and time into regional and national nursing leadership and supporting the development of the youth health nursing sector rather than attaining NP prescription rights. This planned nursing leadership commitment has seen the nurse support and mentor NP candidates within the youth health scope of practice and advance national youth health nursing practice through membership of local, regional and national committees/reference groups. Not seeking prescribing rights was also possible because Standing Orders worked well at Vibe because of the employment of an Adolescent Physician/Paediatrician and general practitioners.

Vibe extended its services during the innovation project. Extensions included opening a new clinic at the Orongomai Marae to improve access to health for Rangitahi (Maori youth), obtaining a contract from the HVDHB to establish clinics at four low decile secondary schools and the establishment of a number of partnerships with other providers to facilitate access to health services 
for local youth. One of the latter partnerships is a Youth Transition Service, funded by the Ministry of Social Development. This service, commenced in 2006, brought about a large increase in youth accessing the clinical service. Its goal is to assist young people between $15-19$ years of age in the transition from school to courses in the community and work. Following the innovation period the HVDHB in its commitment to supporting NPs, assigned funding for Vibe to continue to employ a NP until 2009.

\section{Nursing Practice Development}

The overarching nursing leadership capability brought to the service is informed by a strengths based collaborative and relational philosophical approach (Alcorn, 2007). A youth development philosophy, and application of a model of youth health practice developed as part of her postgraduate study, underpin the nursing practice (Alcorn, 2001). The model, based on the Maori model of health Te Whare Tapu Wha - whanau (family and community), tinana (physical) wairua (spiritual) and hinengaro (mental and emotional) - provides a holistic philosophical approach to guide the clinical practice of the interdisciplinary team. The model includes the $\mathrm{HE}^{2} \mathrm{ADS}^{3}$ (Home environment, Education and employment, Eating, peer related Activities, Drugs, Sexuality, Suicide/ depression and Safety) assessment framework, a USA developed tool for assessing youth health (Goldenring $\&$ Rosen, 2004). The extent of the assessment and details gathered about individuals vary depending on the level of contact with youth. The NP coaches the nurses and doctors who work at Vibe, and whom she supervises, as they apply the model and the assessment tool in clinical practice. The $\mathrm{HE}^{2} \mathrm{ADS}^{3}$ framework identifies risk and resilience factors in order to plan strength-based health care interventions and is widely used within youth practice in New Zealand (Zonneveld, 2007).

Significant in Vibe's expansion is leadership demonstrated through modelling advancing nursing practice and mentoring and teaching others to develop and improve their own practice (Hartranft, Garcia, \& Adams, 2007). A combination of self-referral nurse clinics and health referral/ liaison constitutes over $50 \%$ of the NP's practice. Case management, working intensively with youth in complex situations, is an important practice component. Further, she mentors other clinicians in this role including liaison with the VIBE support team/peer support workers and clinical support for nurses and doctors working as part of the Vibe school-based health service contract. The school personnel liaise with Vibe nurses and doctors as required for immediate practice support/guidance with regard to complex situations. Facilitation of clinical discussion at regular school nurse meetings occurs and the NP's input at Vibe clinical staff meetings supports best practice delivery. Sharing new learning with team members enables consolidation in practice and smoothes the progress of the service.

Liaison with Vibe social support and youth transition staff, Children and 
Young People's Service personnel, midwives, counsellors, pregnancy termination service staff, alcohol and drug services, mental health services, radiologist and laboratory staff forms what is termed a health care mediation role. Health care mediation - Te Takawaenga Hauora - involves accessing community health services as required by young people (Alcorn, 2007). Vibe services seek to provide a first point of contact for individual youth health concerns but youth health clinicians also have a helpline role in accessing additional health services as required, establishing these links with, for and on behalf of young people. Advocacy for young people with regard to outside health provision, supporting seamless health care delivery, and supporting the transition to allied health services form this mediation.

Focus group feedback with both youth clientele and youth peer support workers affirms the responsiveness of the NP to her clients (Connor \& Nelson, 2006; Primary Health Care Nurse Evaluation Team, 2007). In particular, these youth expressed appreciation of her ability to 'engage with clients' and 'go the extra mile' to meet their need and support her clientele to incorporate difficult decisions into their health trajectories. Her attention to clients and to the youth populations generally means that she engages in political endeavours to attain the resources needed to continually transform and advance her practice in relation to community need.

The nurse believed reflective practice, regular attendance at professional workshops and conferences in tandem with the process of applying for NP registration were significant to her professional development. Outside of Vibe, the NP contributes to professional nursing as a visiting lecturer in the Whitireia Community Polytechnic School of Nursing and Health's PHC module. Within this relationship is the organisation of clinical placements for students of nursing from Whitireia. Massey University nursing students are also placed at Vibe. The student placements expanded further in 2006 when Vibe began hosting a new graduate nurse funded by the HVDHB. Although the pathways are different, such developments are not too dissimilar to US developments where clinical leaders are part of educationpractice partnerships (Stanhope \& Pedersen Turner, 2006).

Becoming an NP heightened her profile at the national level with the consequence of other individuals and organisations requesting advice on advancing their progress towards NP status and moves for extending postgraduate nursing education in youth health. National NP involvement includes membership of i) a national steering group to develop "Youth Health Nursing Standards for Effective Practice", ii) the Ministry of Health's Youth Health Advisory Group, and iii) leadership responsibility of a newly established professional society (Society of Youth Health Professionals Aotearoa New Zealand) for nurses, doctors and allied health professionals working in youth health. The broadening sphere of influence in the Vibe service during her journey to $\mathrm{NP}$ and beyond flows into influencing service development. 


\section{Service Development}

Enhancing the expression of clinical leadership capability is the credibility attained in being a fully functional member of the executive team at Vibe. Within this position the nurse clinical leader influences the future direction of the Vibe service as well as facilitates linkages with other local and national agencies associated with youth health and the profession of nursing.

From the platform of clinical leader in 2003, the nurse began work on standards, policies and protocols appropriate to a 'youth friendly' and strengths based Vibe service in order to ensure consistency from all clinical personnel. Liaison with clinical staff from services such as the ' 198 Youth Service' in Christchurch, and others working within youth health, provided a base for the development of policies and protocols for Vibe. Ongoing discussions with youth health clinicians, both locally and nationally, where Vibe's evolving policies and frameworks are shared, continues to support further development of services.

In the Vibe and low decile secondary school clinics there was initiation, development and establishment of standing orders with the medical practitioners. Standing orders allow increased access of young people to the early administration of specified medicines under specified conditions (Ministry of Health, 2002). Specifically, at Vibe, these orders increase timely access to contraception and to the treatment of urinary and sexually transmitted infections. Developing the orders involved working with the Vibe team on clinical, legislative and ethical aspects, particularly concerning the provision of contraceptives to young people under the age of 16 years. The NP believes that the access of young people of any age to contraceptive care, the delivery of clinical care to youth clients and the recording of consultation notes need to reflect a robust and holistic approach to health care provision. Emanating from Vibe nurses using standing orders was the development of youth appropriate pamphlets in collaboration with Whitireia Community Polytechnic on chlamydia, emergency contraception, Depo Provera, and the combined contraceptive pill.

The partnership between Regional Public Health where the low decile school nurses are employed and Vibe requires ongoing liaison to support collaborative clinical support and service delivery at the schools. The introduction of Medtech (electronic health database) across the multiple Vibe clinic sites, including the school clinics, facilitates an exchange of knowledge about clients between personnel thus ensuring a seamless approach to clinical records and audit processes. The school nurses acknowledge respectful, supportive, inspiring, encouraging and inclusive leadership in the NP (Primary Health Care Nurse Evaluation Team, 2007). Moreover, these nurses see that much of the innovative and change agent effectiveness of her role relates to its positioning, outside of traditional health organisations such as those discussed by Weston (2008) as difficult places for achieving transformational leadership. 
During the innovation period (20032006) many of the contracted staff, especially nurses and GPs, became employed by the Vibe Trust Board. This shift created a greater sense of identity as a clinical team supported by the provision of interdisciplinary clinical leadership within the emerging specialist PHC service. The employment at Vibe of a part time Adolescent Physician/Paediatrician in 2005 enhanced the development of interdisciplinary youth health practice. The collaborative and collegial relationship which developed between this medical specialist and the NP improved the level of service delivery offered at Vibe. As has been found in other research (Solheim et al., 2007), the NP's successful leadership of this re-structured multidisciplinary team was achieved as a result of her knowledge, skills and commitment to primary health care.

Support for professional development of Vibe clinicians, and other Vibe staff ensures the strength-based practice is reflected in all aspects of service delivery. It demonstrates how the NP was successful in integrating what Thomas and While (2007) discuss as the horizontal and vertical aspects of the clinical leadership role. Peer support workers at Vibe received mentoring support and guidance with regard to the clinical information they share with clients in one to one and group interventions. An outcome of this mentoring was greater service delivery when, under supervision, the peer support workers promoted the meningococcal $\mathrm{B}$ immunisation campaign and oral health awareness in the Hutt Valley summer festivals attended by young people.
In 2004 the Vibe Service received the Primary Health Care Excellence Award from the HVDHB and in 2005 the NP received a Primary Health Care Innovation Excellence award from the HVDHB for her practice within the Vibe service. These awards are recognition of the innovative culture at Vibe where nursing practice development and service development work in tandem, each contributing to the other.

Involvement with broader policy development is observable in the NP's contributions to regional initiatives of the DHB through membership of the HVDHB PHC Leadership Group and the HVDHB Community and Public Health Advisory Group. Such a platform provides a voice for the special needs of youth in obtaining access to youth friendly services. In using her voice the NP models strategic activities for nurses and other clinicians who contribute to youth health.

In summary, the influence of nursing leadership in Vibe during the innovation and beyond was extensive. Table 1 provides a succinct overview of the extent and complexity of this leadership role.

In understanding this contribution it is important to remember that the NP's previous academic and clinical experience had prepared her to take this opportunity to expand her professional practice. She brought with her the vision and the skills consistent with transformational leadership (Weston, 2008) and in working collaboratively with colleagues achieved transformation of the service. The paper now turns to the NP's own 
Table 1.

\begin{tabular}{|c|c|c|}
\hline Leadership in: & Internal to Vibe & External to Vibe \\
\hline $\begin{array}{l}\text { Practice } \\
\text { Development }\end{array}$ & $\begin{array}{l}\text { - Practice development towards } \\
\text { Nurse Practitioner (NP) } \\
\text { registration } \\
\text { - Coaching \& mentoring } \\
\text { interdisciplinary staff in the } \\
\text { application of youth development } \\
\text { philosophy, youth health model \& } \\
\text { HEADSS assessment } \\
\text { - Health care mediation } \\
\text { - Case management of young } \\
\text { people in complex situations } \\
\text { Teaching nursing students \& new } \\
\text { graduate nurses }\end{array}$ & $\begin{array}{l}\text { - Teaching in undergraduate \& } \\
\text { postgraduate nursing programmes } \\
\text { - Honorary Clinical Associate - } \\
\text { Whitereia Community Polytechnic } \\
\text { - Coaching \& mentoring Vibe/Public } \\
\text { health nurses \& GPs in secondary } \\
\text { schools clinics } \\
\text { - Coaching \& mentoring other youth } \\
\text { health nurses locally \& nationally } \\
\text { - Support for youth health nurses } \\
\text { moving to NP } \\
\text { - Leadership of the Greater Wellington } \\
\text { School Nurses Group }\end{array}$ \\
\hline $\begin{array}{l}\text { Service } \\
\text { Development }\end{array}$ & $\begin{array}{l}\text { Introduction of coherent policy, } \\
\text { protocols \& procedures } \\
\text { - Auditing these protocols } \\
\text { - Development of Personnel } \\
\text { - Development Pathways \& } \\
\text { supporting staff to expand } \\
\text { practice in this framework } \\
\text { - Input into contract construction }\end{array}$ & $\begin{array}{l}\text { Influencing policy development on } \\
\text { HVDHB committee } \\
\text { - Advising national organisations on } \\
\text { supporting development of NPs } \\
\text { - Ministry of Health - Youth Health } \\
\text { Advisory Group member }\end{array}$ \\
\hline
\end{tabular}

voice as she reflects on her most significant moments of her role as clinical leader at Vibe.

\section{Significant moments - reflections from the Vibe Nurse Practitioner}

Significant moments for me evolved from my opportunity to play a part in establishing a strength-based model of nursing practice delivery while embarking upon a journey towards becoming a NP (Youth Health). The need for youth health services is apparent in New Zealand (Clendon, 2005; Clendon \& Krothe, 2004; Dutton, 2006).

The successful establishment of Vibe-school-based services at the four schools and the continued high utilisation of these services are significant. My role in supporting the practice of the Vibe nurses and doctors working in these schools influences practice and contributes to improved outcomes for the youth served. The school health services are highly utilised and the greater clinical capacity is desirable to address the health needs reflected within the school communities. The services will be enhanced through new funding allocated by the Government in 2008 to support the development of health services in teen parent units, alternative education units and decile 1 -3 schools (1.0 FTE registered nurse: 750 students) over a three year period. This new funding will also enable registered nurses trained in youth health practice to undertake nursing assessments on all year 9 students in decile 1-3 schools. This investment in youth health service nationally will support early intervention across a range of health needs and has the 
potential to reduce inequalities and improve youth health outcomes.

Mentoring colleagues is a nourishing professional experience. At one level, this involves new graduate nurses entering the youth health specialty practice area. At another level are those working towards NP registration. Supporting the professional development of nurses on the NP pathway, and advancing youth health specialty practice in New Zealand is highly rewarding. The current registration of three NPs with the Youth Health scope of practice is very encouraging for this emerging nursing speciality.

Another highlight was the successful establishment, in partnership with BirthEd - HVDHB birth education contractor, of Vibe's youth appropriate antenatal education. A focus group, facilitated by me at the local teenage parent school in October 2003, provided information that mainstream antenatal education classes were not meeting the needs of young women and their support people. The reasons given for this gap were social stigma, vulnerability, and perceived judgemental attitudes. A collaborative partnership between BirthEd and Vibe began the development of youth appropriate antenatal classes in 2005. Between 2005 and 2008 over 200 young women participated in this programme and evaluation information indicates that many of these would recommend this programme to others. Positive outcomes measured in attendance numbers, course completion, client satisfaction, breastfeeding rates have been markers of this experience. Enhancing my practice is the personal awareness I gained from observing individuals and the group's journey towards overcoming societal judgement and vulnerability, and addressing their parenting role positively.

Overall, participating in the innovation gave me the opportunity to develop a vision for youth health nursing that until then I was only theorising about. Of particular significance was the attendance at the annual workshops organised by the evaluation team and attended by representatives of all 11 innovation projects. These workshop interludes provided affirmation of my efforts and ideas and provided a nurturing space to connect with national nursing leaders. They were an opportunity to discuss innovative practice and rekindle my energy with regard to youth health service development.

Reflecting on my practice is integral to advancement in my practice journey. So also is my preference to express these reflections in poetry. The following poem is an example of this expression. It mirrors the stories of personal health challenges that I hear in my everyday practice.

\section{Relief of Grief}

Disclosure, a frighteningly lucid narrative why does it happen, a breach of trust a child's body taken in lust powers realm gathers memories offensive

an embryo of self, dust to dust pulled to the underworld, Hades crib

Clamours for relief of grief

Silver and gold tinkling jingles softly tended 
body adornment, cryptic messages bright as due

tongue rolls hiding bolt and screw majestic pain defiantly extended metallic glory, medals with strength imbue

loss resonates pain, self-inflicted

Take it on the chin with pin

Remember latent glimpses of adults tender

quest for loves touching embrace consumes

sensual moments exchanged in smoke filled rooms

vineyards yield anaesthetises unplanned surrender

menses late by days signals life in womb wondrous creation, conceals hidden gender

What to do with you?

Chanceful risk understands not how generations slumber

romantic fiction counterpoints with shame

to have and hold or vanquish bairn sleep not nor visit images of cherubim without number

eyes weep tears of grief and pain eloquent answers deny, infinite grace to stumble

Wisely hold this story told

\section{Conclusion}

Innovations funding provided the opportunity to develop the potential of clinical nursing leadership in a small multidisciplinary PHC service situated in a New Zealand youth health service. The coming together of a DHB supportive of youth health development, and the VIBE service team and clinical leader each bringing their own personal strengths, achieved a transformation of the service during the funding period and beyond. In explicating the work of the clinical leader in such a context unfettered by hierarchical and bureaucratic hurdles there is evidence of the contribution that can be made through clinical leadership. Such a transformative model of working can provide impetus and inspiration to others seeking changes in the PHC services in which they are embedded.

\section{Acknowledgements}

We would like to acknowledge Trish Wright (HealthRight, Waikato Primary Health), Dr Jacqueline Cumming and Susan Buckley (Health Services Research Centre, Victoria University of Wellington) and Dr Jan Pearson (Cancer Society of New Zealand) for their contributions to the Primary Health Care Nursing Evaluation.

\section{References}

Abbott, S. (2007). Leadership across boundaries: A qualitative study of the nurse consultant role in English primary care. Journal of Nursing Management, 15, 703-710.

Alcorn, G. (2001). Giving voice to school nursing as a primary health care specialty. Unpublished master's thesis, Victoria University of Wellington, Wellington.

Alcorn, G. (2007). The youth health speciality in New Zealand: Collaborative practice and future development. New Zealand Family Physician, 34(3), 162-167.

Clendon, J. (2005, May). Nursing in schools: Time for a change. New Zealand Nursing Review, 6, 16. 
Clendon, J., \& Krothe, J. (2004). The nurse-managed clinic: An evaluative study. Nursing Praxis in New Zealand, 20(2), 15-23.

Connor, M., \& Nelson, K. (2006). 'It's about us': An evaluation of Vibe youth health services (An unpublished report for Vibe).

Dutton, J. (2006). Primary health care for youth in New Zealand. Are current health strategies working? Nursing Praxis of New Zealand, 22(2), 12-20.

Goldenring, J. M., \& Rosen, D. S. (2004). Getting into adolescent heads: An essential update. Contemporary Paediatrics, 21(1), 64-90.

Hartranft, S., Garcia, T., \& Adams, N. (2007). Realizing the anticipated effects of the clinical nurse leader role. Journal of Nursing Administration, 36, 446-449.

Hyrkäs, K., \& Dende, D. (2008). Clinical nursing leadership - perspectives on current topics. Journal of Nursing Management, 16, 495-498.

MacKay, B. (2002). Leadership development: Supporting nursing in a changing primary health care environment Nursing Praxis of New Zealand, 18(2), 24-32.

McMurray, A. (2007). Leadership in primary health care: An international perspective. Contemporary Nurse, 26(1), 30-36.

Ministry of Health. (1995). Nursing leadership development. Wellington: Author.

Ministry of Health. (2001). The primary health care strategy. Wellington: Author.

Ministry of Health. (2002). Guideline for the development and operation of Standing Orders: Author.

Nursing and Midwifery Workforce Strategy Group. (2006). Nursing workforce strategy. Retrieved September 15, 2008, from http://www.dhbnz.org.nz/Site/Future_Workforce/ Default.aspx

Nursing Council of New Zealand. (2004). Scopes of practice. Retrieved February 2, 2005, from www.nursingcouncil.org.nz/scopes.html

Primary Health Care Nurse Evaluation Team. (2007). The evaluation of the eleven primary health care nursing innovation projects: A report to the Ministry of Health by the Primary Health Care Nurse Innovation Evaluation Team. Retrieved October 1, 2007, from www. moh.govt.nz

Solheim, K., McElmurry, B. J., \& Kim, M. J. (2007). Multidisciplinary teamwork in US primary health care. Social Science \& Medicine, 65, 622-634.

Stanhope, M., \& Pedersen Turner, L. (2006). Diffusion of the clinical nurse leader innovation. Journal of Nursing Administration, 36, 385-389.

Thomas, P., \& While, A. (2007). Should nurses be leaders of integrated health care? Journal of Nursing Management, 15, 643-648.

Weston, M. J. (2008). Transformational leadership at a national perspective. Nurse Leader, $6(4), 41-45$.

Zonneveld, R. (2007). Evolve Wellington Youth Service: A community service developed by and for young people. New Zealand Family Physician, 34(3), 173-176. 